\title{
Glycolic acid peel therapy - a current review
}

This article was published in the following Dove Press journal:

Clinical, Cosmetic and Investigational Dermatology

8 November 2013

Number of times this article has been viewed

\section{Jaishree Sharad}

Skinfiniti Aesthetic Skin and Laser Clinic, Mumbai, India
Correspondence: Jaishree Sharad Skinfiniti Aesthetic Skin \& Laser Clinic, 202, Sun Vihar, Plot I53, Sector I2, Vashi, Navi Mumbai, Maharashtra, India, 400703

Tel +9l 9820058598

Email jaishree19@gmail.com
Abstract: Chemical peels have been time-tested and are here to stay. Alpha-hydroxy peels are highly popular in the dermatologist's arsenal of procedures. Glycolic acid peel is the most common alpha-hydroxy acid peel, also known as fruit peel. It is simple, inexpensive, and has no downtime. This review talks about various studies of glycolic acid peels for various indications, such as acne, acne scars, melasma, postinflammatory hyperpigmentation, photoaging, and seborrhea. Combination therapies and treatment procedure are also discussed. Careful review of medical history, examination of the skin, and pre-peel priming of skin are important before every peel. Proper patient selection, peel timing, and neutralization on-time will ensure good results, with no side effects. Depth of the glycolic acid peel depends on the concentration of the acid used, the number of coats applied, and the time for which it is applied. Hence, it can be used as a very superficial peel, or even a medium depth peel. It has been found to be very safe with Fitzpatrick skin types I-IV. All in all, it is a peel that is here to stay.

Keywords: acne scar, melasma, photoaging, chemical peel, alpha-hydroxy peel

\section{Introduction}

Alpha-hydroxy peels have been popular in dermatological practice for years and are well-established. They have practically no downtime, and are usually superficial or medium depth peels. They have therapeutic as well as cosmetic benefits when used on skin. ${ }^{1}$ Glycolic acid (GA) obtained from sugarcane is used in the most common alpha-hydroxy acid peel. ${ }^{2}$

GA has the smallest molecular weight amongst all the alpha-hydroxy acids. It penetrates skin easily, making it a popular peel agent. ${ }^{3} \mathrm{GA}$ has two carbon atoms: one carbon atom is with a carboxyl group and the other carbon atom is with a hydroxyl group. GA is extremely hydrophilic. The $\mathrm{pH}$ of a non-buffered solution ranges from 0.08-2.75. ${ }^{4}$ Previous authors have recommended the use of a buffered or partially neutralized GA, which is safer than free GA. ${ }^{5}$ Glycolic acid peels are commercially available as free acids, partially neutralized (higher $\mathrm{pH}$ ), buffered, or esterified solutions. ${ }^{6}$ They are available in various concentrations ranging from $20 \%-70 \%$. The higher the concentration and lower the $\mathrm{pH}$, the more intense the peeling will be. ${ }^{7}$ In general, gel formulations have a slower penetration time and are easier to control. ${ }^{8}$

Fabbrocini, in 2009, classified glycolic peels as: very superficial (30\%-50\% GA, applied for 1-2 minutes); superficial (50\%-70\% GA, applied for 2-5 minutes); and medium depth (70\% GA, applied for 3-15 minutes). ${ }^{8}$ GA peels have antiinflammatory, keratolytic, and antioxidant effects. GA targets the corneosome by enhancing breakdown and decreasing cohesiveness, causing desquamation. ${ }^{9}$ The intensity of 
GA peel is determined by the concentration of the acid. ${ }^{10}$ GA peels need to be properly neutralized in order to stop acidification of the skin. ${ }^{11}$

\section{Material and methods}

\section{Application methods}

Before starting a series of GA peels, the status of the skin should be assessed for the occurrence of any dry or scaly patches on skin, open sores that may have become acidified through the use of GA/tretinoin creams. ${ }^{12}$ Priming the skin with hydroquinone, or topical retinoids, before performing a peel has been found to increase peel efficacy and reduce the risk of postinflammatory hyperpigmentation. ${ }^{13}$ After the skin has been cleansed and degreased, GA solution is applied using cotton buds or a brush in a sequential manner starting from the forehead to the left cheek, chin, right cheek to cover the entire face. Care is taken to protect the eyes and the corners of the nose and lips. The peel is neutralized within 3-5 minutes, or when uniform erythema is seen. If frosting is observed in any particular area before the set time or end-point, it is important to neutralize the peel immediately. It is always better to start with a low concentration (20\% GA) and increase the concentration and application time during subsequent sessions. ${ }^{3}$ Peeling is repeated once every 15 days for 4-6 months until the desired result is achieved. ${ }^{14}$

\section{Mechanism of action}

Glycolic acid peels have antiinflammatory, keratolytic, and antioxidant effects. GA targets the corneosome by enhancing breakdown and decreasing cohesiveness, causing desquamation. ${ }^{9}$ The intensity of peel is determined by the concentration of the acid, the vehicle used to carry it, the amount of acid applied, and the technique used. ${ }^{10}$

GA peels need to be properly neutralized to stop acidification of the skin. Applying acid to the skin saturates the ability of cells to resist acidification; excess acid must be neutralized to avoid burning. Alpha-hydroxy acid peels can be neutralized with water or with basic solutions, such as ammonium salts, sodium bicarbonate, or sodium hydroxide. ${ }^{11}$

\section{Indications}

Acne, acne scars, melasma, postinflammatory hyperpigmentation, photoaging, and seborrhea are indications for chemical peeling. ${ }^{15-19}$ GA may be used in acne also to normalize keratinization and increase epidermal and dermal hyaluronic acid and collagen gene expression. ${ }^{20}$

\section{Acne and acne scars}

In a study by Wang et al, Asian patients with skin type IV with acne were treated with $35 \%$ GA and $50 \%$ GA peels, once in 3 weeks for 10 weeks. There was significant resolution of comedones, papules, and pustules. The skin texture improved and follicular pore size reduced. Most of the patients were found to have brighter and lighter looking skin. Consistent and repetitive treatment with GA was needed for the apparent improvement of acne scars and cystic lesions. Only a small percentage of patients (5.6\%) developed side effects, in the form of postinflammatory hyperpigmentation, mild skin irritation, and exacerbation of herpes simplex infection. Hence, GA peels were found to be ideal as an adjunctive treatment for acne. ${ }^{21}$

In another study by Atzori et al, 70\% GA was used in comedonic acne, papulopustular, and nodular/cystic acne. While comedones improved rapidly, papulopustular acne improved after six peel sessions, and nodulocystic lesions required ten sessions at three-week intervals. A significant improvement of coexisting post-acne superficial scarring was noted. Patient tolerance and compliance were both found to be excellent. ${ }^{22}$

Kim et al did a comparative study using $70 \%$ GA and Jessner's Solution (resorcinol 14\%, lactic acid 14\%, and salicylic acid 14\%, in an alcohol base), respectively, in two groups of patients with acne. Three peel sessions were done for each group. Though acne improved in both to the same extent, there was more exfoliation seen in the Jessner's Solution group. Hence, GA was found to be the better tolerated of the two peels for the treatment of acne in Asian patients. ${ }^{23}$

Grover and Reddu conducted a study of 41 patients with Fitzpatrick Skin Type III-V, of whom 16 patients had acne. Initially, they used $10 \%$ GA for a period of 1-2 minutes, then gradually increased the duration to 5 minutes and concentration to $30 \%$ GA. All patients had greasy skin with high sebaceous activity. The severity of acne was graded on a four-point scale, which revealed that mild to moderate acne was seen in eight patients, severe acne in seven patients and one patient had nodulocystic acne. Apart from comedones, papules, and pustules, a significant number of patients had scarring and pigmentation. After undergoing peels with GA, the therapeutic response was good in $75 \%$ of patients, on the basis of both patient and observer assessments. Patients with postinflammatory hyperpigmentation and scarring showed excellent improvement. Significant decrease in the number of comedones and papulopustules was observed in patients with mild to moderate acne. However, the patient with nodulocystic acne lesions did not respond well to therapy. Compared to the $90 \%$ response seen by Wang et al, ${ }^{21} 75 \%-78 \%$ of patients 
with acne, excepting the patient with nodulocystic acne, showed a good-to-fair response with GA peels. ${ }^{24}$

Kessler et al compared 30\% GA versus 30\% salicylic acid (SA) peels in 20 patients with mild to moderate acne, using a split-face design. Peels were performed every 2 weeks for a total of six treatments. Both peels improved acne. However, the authors found that the SA peel had better sustained efficacy (ie, number of acne lesions, and improvement rating by blinded evaluator) and fewer side effects than GA, presumably due to the greater lipophilicity of SA. The authors agreed with the impression that SA peels are better tolerated than GA peels in acne patients. ${ }^{25}$

A study by Erbağci and Akçali concluded that a 70\% GA peel performed every 2 weeks resulted in significant improvement in atrophic acne scarring, as compared to $15 \% \mathrm{GA}$ cream used daily. In one group of 23 patients, GA peels were performed bi-weekly with increasing application time and acid concentration from $20 \%-70 \%$. A second group of 20 patients was instructed to use $15 \%$ GA cream twice daily for a period of 24 weeks. It was seen that six patients, using $70 \%$ GA, showed significantly better results than daily use of $15 \%$ GA for 24 weeks $(P<0.05)$. Furthermore, apparently good responses were observed in the peel group only $(P<0.01){ }^{26}$

In the author's experience, GA peels are excellent for use on Indian skin. Patients with Fitzpatrick's skin types III-IV with post acne pigmentation are treated with $20 \%$ GA peel for the first three sessions. The concentration of GA is then increased to $35 \%$ in the subsequent five to six sessions. There is an interval of 15 days between sessions. Patients respond well, with clearance of lesions, in

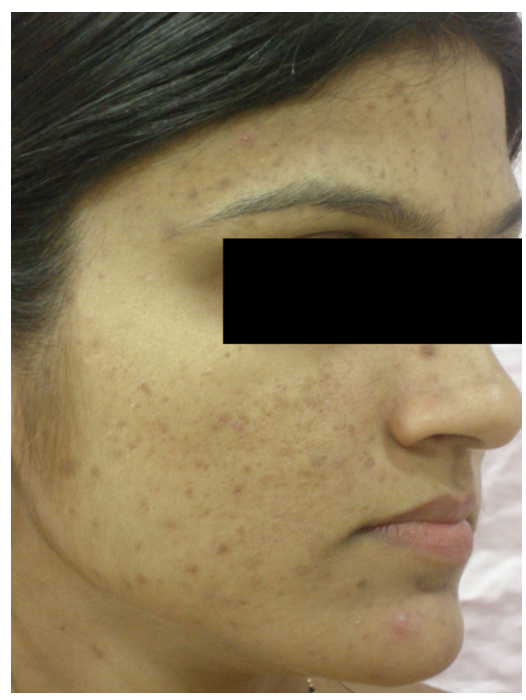

Figure I Post-acne scars and pigmentation before glycolic acid peel.

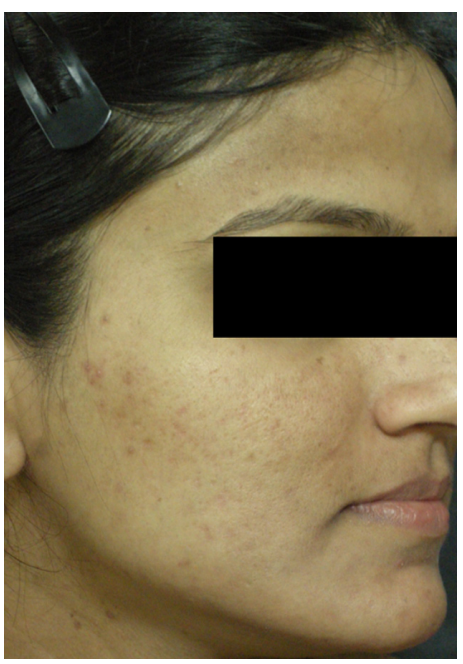

Figure 2 Post-acne scars and pigmentation after eight sessions of glycolic acid peel.

around eight to ten sessions, without any adverse effects (Figures 1 and 2).

\section{Melasma}

Lin and Tham studied the use of GA peels in ten Asian women. Concentrations of $20 \%-70 \%$ GA were administered every 3 weeks, either alone or in combination with a topical regimen of $2 \%$ hydroquinone plus $10 \%$ GA. There was significant improvement $(P<0.06)$ in melasma and fine facial wrinkling in patients who received the combination of creams and peeling. ${ }^{27}$

Kalla et al did a comparative study of $55 \%-75 \%$ GA versus $10 \%-15 \%$ trichloroacetic acid (TCA) peels in 100 patients with recalcitrant melasma. The peels were conducted at 15-day intervals in both groups. It was seen that the response to TCA was rapid, and produced better results than GA. However, relapse was more common in the TCA group $(25 \%)$ than in the GA group $(5.9 \%){ }^{28}$

In a study by Javaheri et al, peeling was performed upon 15 Indian females with melasma, using 50\% GA, once-monthly for 3 months. An improvement in Melasma Area Severity Index (MASI) score was observed in 91\% of patients $(P<0.01)$. A better response was seen in patients with epidermal melasma, compared to those with mixed melasma $(P<0.05){ }^{29}$

In a study by Sarkar et al, 20 Indian patients received serial GA peels $(30 \%$ GA for the first three sittings; $40 \%$ GA for the next three sittings), combined with the modified Kligman's formula ( $2 \%$ hydroquinone, $0.025 \%$ tretinoin, and $1 \%$ mometasone). A further 20 Indian patients received only the modified Kligman's formula, with no peeling. In both groups, 
a significant decrease in the MASI score was observed from baseline to 21 weeks $(P<0.001)$. However, the GA peel group showed more rapid and greater improvement $(P<0.001) .{ }^{30}$

A comparative study by Hurley et al was done on 21 Hispanic women with bilateral, epidermal, and mixed melasma to assess the efficacy of $4 \%$ hydroquinone cream versus $4 \%$ hydroquinone cream combined with GA peels. Patients received GA peels ( $20 \%-30 \%$ GA) every 2 weeks to one side of the face only, in addition to twice-daily application of $4 \%$ hydroquinone cream to the other side of the face. Pigmentation was measured objectively using a Mexameter ${ }^{\circledR}$ (Courage + Khazaka electronic GmbH, Cologne, Germany) and the MASI, and measured subjectively using a linear analog scale and physician and patient global evaluation. Both sides of the face showed a reduction of pigmentation, and there was no significant difference. ${ }^{31}$

The concentration of GA used by Hurley et al was low $(20 \%-30 \%)$, compared to the $30 \%-40 \%$ GA used by Sarkar et al. ${ }^{32}$ This could be a reason for the difference in the results they observed.

In a study by Khunger et al, patients with melasma were treated with a $70 \%$ GA peel on one half of the face, while the other half was treated with a $1 \%$ tretinoin peel. A significant decrease in the modified MASI score was observed on both facial sides from baseline to 6 weeks, and then from 6 to 12 weeks $(P<0.001) .{ }^{33}$ A study by Kligman found the two peels to be equally effective and well tolerated. ${ }^{34}$

In 15 cases of melasma (epidermal: $80 \%$; dermal: 13.3\%; and mixed: $6.6 \%$ ), 52.5\% GA concentration was applied for 3 minutes. There was good to fair response in patients with epidermal and mixed melasma, while no significant improvement was seen in dermal melasma. ${ }^{24}$

Serial GA peels (from 35\%-50\%, and 70\% every second peel) plus combination topical therapy (azelaic acid and adapalene) in 28 women with melasma found better results in the group receiving chemical peel plus topical therapy $(P=0.048)$, but only when the GA concentration was $50 \%$ or higher. ${ }^{35}$

In another study, a triple combination cream consisting of fluocinolone acetonide $0.01 \%$, hydroquinone $4 \%$, and tretinoin $0.05 \%$ was used in an alternating sequential treatment pattern, cycling with a series of GA peels, for the treatment of moderate to severe melasma. Spectrometry measurement of the difference in melanin between involved and uninvolved skin confirmed that hyperpigmentation was significantly reduced at 6 and 12 weeks, compared with baseline $(P<0.001)$, with evaluations showing improvement of $90 \%$ or more by week $12 .{ }^{36}$
In another study, 10 patients with melasma were treated with a triple combination of tretinoin $0.05 \%$, hydroquinone $4 \%$, and mometasone furoate $0.1 \%$. Serial GA peels were performed at three-weekly intervals, with application times from 2-6 minutes, depending on tolerance and erythema. Glycolic acid of $57 \%$ concentration, with $55 \%$ free acid, and $\mathrm{pH} 2.3$ was used on the face in gradually increasing durations of application. Significant reduction in melasma was noted after four peels. One patient developed irritation and hyperpigmentation, while one developed persistent erythema. No other side effects were reported..$^{37,38}$

In a comparative study of $10 \%-20 \%$ TCA versus $20 \%-35 \%$ GA peels for the treatment of melasma, similar improvement was seen with both peels. However, the GA peel was seen to be associated with fewer side effects than the TCA peel, and gave the added benefit of facial rejuvenation. ${ }^{39}$

In another similar study of $15 \%$ TCA peel versus $35 \%$ GA peel for the treatment of melasma, there was no statistically significant difference in efficacy. Both peels significantly reduced MASI scores, and both were found to be equally effective in the treatment of melasma. It was also seen that adverse effects were more common with TCA than with GA peels. ${ }^{40}$

\section{Postinflammatory hyperpigmentation}

In a pilot study by Burns et al, postinflammatory hyperpigmentation was treated with a series of GA peels in skin types IV-VI. No adverse effects were reported in dark skin, and the GA peel proved to be efficacious. ${ }^{41}$ This echoed the study by Grover and Reddu, in which skin types III-V showed overall improvement of skin texture in almost all patients. ${ }^{24}$

In the author's experience with cases of postinflammatory hyperpigmentation in skin types III and IV, a series of $35 \%$ GA peels has produced good results. All patients are instructed to use sunscreen before and after the peel. Priming is done with a combination of mometasone and tretinoin $0.025 \%$ creams for 3 weeks before the peel. The patient is asked to stop the creams 1 week before the peel. Post-peel care, in the form of sun protection, and avoidance of facial scrubs, steam, and sauna is advised. Complete resolution of postinflammatory hyperpigmentation is commonly seen after six to eight peel treatments (Figures 3 and 4).

\section{Photodamage}

Photodamage, in the form of dyschromias, actinic keratoses, solar lentigines, and fine wrinkling has also shown improvement with a combination of GA and TCA peels. ${ }^{42,43}$ In a study 


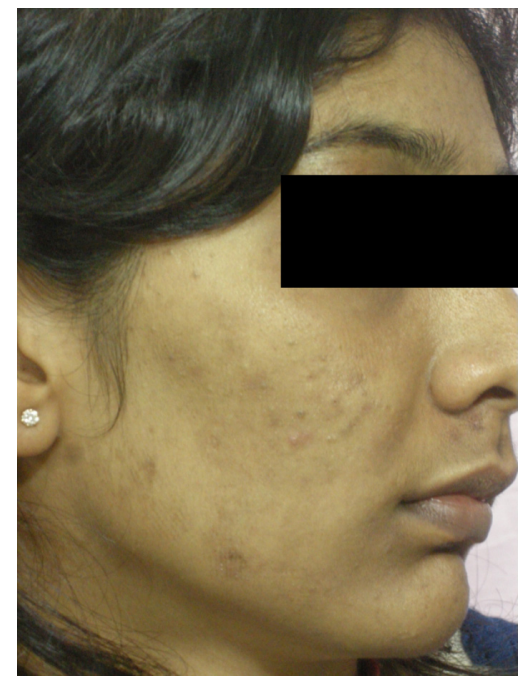

Figure 3 Postinflammatory hyperpigmentation before glycolic acid peel.

of $50 \%$ GA peels by Newman et al, there was improvement in mild photoaging of skin. Other significant improvements were noted, including decreases in rough texture and fine wrinkling, fewer solar keratoses, and slight lightening of solar lentigines. Histologic analysis showed thinning of the stratum corneum, granular layer enhancement, and epidermal thickening. Some specimens showed an increase in collagen thickness in the dermis. ${ }^{44,45}$ Glycolic acid peels do not affect deep wrinkles or deep pigmentations. ${ }^{3}$

\section{Combination treatments}

GA peels have frequently been combined with other peels and treatments, to give better results. In a study by the author, microneedling was combined with $35 \%$ GA peel to treat acne scars in patients with skin type III-IV. Microneedling was

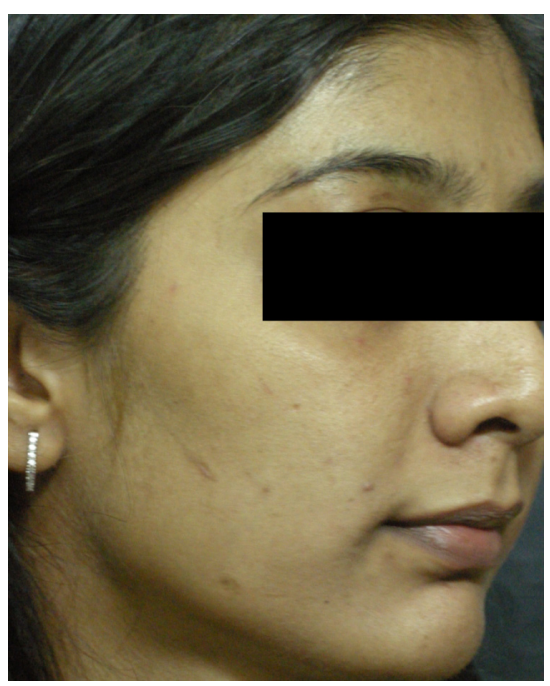

Figure 4 Postinflammatory hyperpigmentation after glycolic acid peel. performed six-weekly, and 35\% GA peel was performed 3 weeks after each microneedling session. There was significant improvement in superficial and moderately deep atrophic box scars and rolling scars. In addition, there was improvement in skin texture and reduction in post-acne pigmentation. ${ }^{46}$

GA and TCA peels are performed sequentially in cases of postinflammatory hyperpigmentation, post-acne pigmentation, and melasma. This combination has been found to produce a deeper and more uniform peel than TCA used alone. $^{47}$

Combining Jessner's Solution and GA for the treatment of photoaged skin, actinic keratoses, and rhytides resulted in a uniform GA peel, but the risk of overpeel and scarring are high, especially in dark-skinned individuals. ${ }^{48-50}$ GA has been combined with 5-fluorouracil to treat actinic keratosis. Pretreatment of the skin with 5-fluorouracil 5\% increases the efficacy of the treatment and shortens the healing time. ${ }^{51}$

GA peeling has also been used in combination with microdermabrasion, for the treatment of acne vulgaris and superficial acne scars, in order to increase treatment efficacy and achieve treatment goals within a shorter time. Alpha-hydroxy acid peels decrease corneocyte cohesion, making the abrasion more efficient. ${ }^{3}$ However, combining GA peels with microdermabrasion at the same session could lead to postinflammatory hyperpigmentation in skin types III-VI. Therefore, care should be taken with darker skin types. ${ }^{52}$ Briden et al reported good patient satisfaction when using superficial GA peels with microdermabrasion for photoaging. ${ }^{53}$

In a study on photodamaged skin, an intense light that combined a narrow-band $(405-420 \mathrm{~nm})$ blue light (antiinflammatory) emission with a near-infrared (850-890 nm) emission was applied to the skin. Concomitant glycolic peels were performed, and daily Vitamin C cream was given. Results showed significant improvements in pore size, rhytids, and radiance. ${ }^{54} \mathrm{GA}$ peeling is also combined with Vitamin $\mathrm{C}$ in cases of melasma and postinflammatory hyperpigmentation. In a patient with melasma, 70\% GA peeling was performed on one side of the face, along with iontophoresis with nanosome vitamin C; 70\% GA peeling alone was performed on the other side of the face. It was seen that both sides improved, but that the side treated with iontophoresis and nanosome Vitamin C showed better results. ${ }^{55}$ Superficial GA peels can also be used with botulinum toxin and fillers in order to obtain overall improvement in wrinkles, skin tone, texture, radiance, and clarity. In one study, the interval between peels and fillers was 1 week. The peel was 
Table I Classification of glycolic acid peels

\begin{tabular}{lll}
\hline Type & Concentration & Duration of application \\
\hline Very superficial & $30 \%-50 \%$ & $1-2$ minutes \\
Superficial & $50 \%-70 \%$ & $2-5$ minutes \\
Medium-depth & $70 \%$ & $3-15$ minutes \\
\hline
\end{tabular}

Note: Data from Fabbrocini et al. ${ }^{8}$

administered after injecting botulinum toxin during the same visit, or the procedures were separated by one or more days to minimize the potential for side effects. ${ }^{8,56,57}$

\section{Side effects}

The GA peel is time-tested. Proper selection of patients, timing of peel, and neutralization on-time should ensure good results and no side effects. Superficial peels using alphahydroxy acids increase the epidermal activity of enzymes, leading to epidermolysis and exfoliation. ${ }^{10}$

The minor side effects reported are: erythema, stinging sensation, sensation of pulling of facial skin, mild burning, and transient postinflammatory hyperpigmentation. Unbuffered GA can cause erosive blisters and scarring. ${ }^{40,58}$ In rare cases, hypopigmentation, persistent erythema, and flare-up of pimples have been reported. . $^{2,36,59}$

Epidermolysis may occur if the patient has used topical retinoids, anti-acne creams, or skin lighteners in days prior to the peel. Other causes are excessive facial scrubbing before the peel, and pre-existing dry skin. It is important to stop priming agents around 1 week before the peel. Sun exposure after the peel may cause postinflammatory hyperpigmentation. ${ }^{60}$ There has been one report of contact urticaria with a GA peel. ${ }^{61}$

\section{Discussion}

Glycolic acid is the alpha-hydroxy acid used most commonly by dermatologists for chemical peeling. It is used in concentrations from $20 \%-70 \%$ in all skin types. It should be used in lower concentrations initially, and then the strength of the peel should be gradually increased in subsequent sessions. There should be a minimum interval of 2 weeks between two treatment sessions. Peel neutralization is extremely important and it depends on erythema seen. However, in dark skin, it may be difficult to appreciate erythema. In such cases, it is better to time the peel between 3-5 minutes and judge the desired end-point depending on the time. ${ }^{2}$ The longer the duration, the deeper the depth of the peel. Pre-peel priming and post-peel care are equally important. Depending on the condition, tretinoin, kojic acid, and hydroquinone can be used for pre-peel priming. In order to avoid postinflammatory hyperpigmentation and scarring in dark-skinned patients, it is important to avoid facial scrubs, depilatory creams, waxing, bleaching, microdermabrasion, and laser hair removal for at least 1 week before the procedure. After the peel, the patient should be advised to avoid sun exposure, facial scrubs, loofahs, picking at the peeling skin, taking steam inhalation, and using creams that cause exfoliation. The patient must be instructed to use a broad spectrum sunscreen during the day, and a moisturizer at night. The GA peel is safe for a correctly selected patient when the correct technique is used. It does not produce any systemic toxicity. It can be combined with other treatment modalities for better cosmetic outcomes.

\section{Conclusion}

The GA peel has proven to be efficacious through the years. Combining the GA peel with other treatment modalities may become more common in future. However, the GA peel is here to stay, as it is a simple, evidence-based, result-oriented, and cost-effective procedure.

\section{Disclosure}

The author reports no conflicts of interest in this work.

\section{References}

1. Tung RC, Bergfeld WF, Vidimos AT, Remzi BK. Alpha-hydroxy acidbased cosmetic procedures. Guidelines for patient management. Am J Clin Dermatol. 2000;1(2):81-88.

2. Khunger N. Step by Step Chemical Peels. Delhi, India: Jaypee Brothers; 2010:69.

3. Landau M. Chemical peels. Clin Dermatol. 2008;26(2):200-208.

4. Roberts WE. Chemical peeling in ethnic/dark skin. Dermatol Ther. 2004;17(2):196-205.

5. Becker FF, Langford FP, Rubin MG, Speelman P. A histological comparison of $50 \%$ and $70 \%$ glycolic acid peels using solutions with various pHs. Dermatol Surg. 1996;22(5):463-465.

6. Ditre CM. Glycolic acid peels. Dermatol Ther. 2000;13(2):165-172.

7. Fischer TC, Perosino E, Poli F, Viera MS, Dreno B; Cosmetic Dermatology European Expert Group. Chemical peels in aesthetic dermatology: an update 2009. J Eur Acad Dermatol Venereol. 2010;24(3): 281-292.

8. Fabbrocini G, De Padova MP, Tosti A. Chemical peels: what's new and what isn't new but still works well. Facial Plast Surg. 2009;25(5): 329-336.

9. Fartasch M, Teal J, Menon GK. Mode of action of glycolic acid on human stratum corneum: ultrastructural and functional evaluation of the epidermal barrier. Arch Dermatol Res. 1997;289(7):404-409.

10. Brody HJ. Chemical Peeling. St Louis: Mosby-Year Book; 1992.

11. Deprez P. Textbook of Chemical Peels: Superficial, Medium and Deep Peels in Cosmetic Practice. London: Informa Healthcare; 2007.

12. Rullan P, Karam AM. Chemical peels for darker skin types. Facial Plast Surg Clin North Am. 2010;18(1):111-131.

13. Garg VK, Sarkar R, Agarwal R. Comparative evaluation of beneficiary effects of priming agents ( $2 \%$ hydroquinone and $0.025 \%$ retinoic acid) in the treatment of melasma with glycolic acid peels. Dermatol Surg. 2008;34(8):1032-1039; discussion 1340.

14. Monheit GD. Skin preparation: an essential step before chemical peeling or laser resurfacing. Cosmet Dermatol. 1996;9:13-14.

15. Bryan CP. Ancient Egyptian medicine: The Papyrus Ebers. Chicago, IL: Ares; 1974:32. 
16. Handog EB, Datuin MS, Singzon IA. Chemical peels for acne and acne scars in Asians: evidence based review. J Cutan Aesthet Surg. 2012;5(4):239-246.

17. Monheit GD, Kayal JD. Chemical peeling. In: Nouri K, Leal-Khouri S, editors. Techniques in Dermatologic Surgery. St Louis, MO: Elsevier; 2003:233-244.

18. Rubin MG, Tung R, editors. Procedures in Cosmetic Dermatology Series: Chemical Peels. St Louis, MO: Elsevier; 2006:1-12.

19. Savant SS. Superficial and medium depth chemical peeling. In: Savant SS, editor. Textbook of Dermatosurgery and Cosmetology. 2nd ed. Mumbai, India: ASCAD; 2005. p. 177.

20. Bernstein EF, Lee J, Brown DB, Yu R, Van Scott E. Glycolic acid treatment increases type I collagen $\mathrm{mRNA}$ and hyaluronic acid content of human skin. Dermatol Surg. 2001;27(5):429-433.

21. Wang CM, Huang CL, Hu CT, Chan HL. The effect of glycolic acid on the treatment of acne in Asian skin. Dermatol Surg. 1997;23(1): 23-29.

22. Atzori L, Brundu MA, Orru A, Biggio P. Glycolic acid peeling in the treatment of acne. J Eur Acad Dermatol Venereol. Mar 1999;12(2):119-122.

23. Kim SW, Moon SE, Kim JA, Eun HC. Glycolic acid versus Jessner's solution: which is better for facial acne patients? A randomized prospective clinical trial of split-face model therapy. Dermatol Surg. 1999;25(4)270-273.

24. Grover C, Reddu BS. The therapeutic value of glycolic acid peels in dermatology. Indian J Dermatol Venereol Leprol. 2003;69(2): 148-150.

25. Kessler E, Flanagan K, Chia C, Rogers C, Glaser DA. Comparison of alpha- and beta-hydroxy acid chemical peels in the treatment of mild to moderately severe facial acne vulgaris. Dermatol Surg. 2008;34(1):45-50; discussion 51 .

26. Erbağci Z, Akçali C. Biweekly serial glycolic acid peels vs long-term daily use of topical low-strength glycolic acid in the treatment of atrophic acne scars. Int J Dermatol. 2000;39(10):789-794.

27. Lim JT, Tham SN. Glycolic acid peels in the treatment of melasma among Asian women. Dermatol Surg. 1997;23(3):177-179.

28. Kalla G, Garg A, Kachhawa D. Chemical peeling-glycolic acid versus trichloroacetic acid in melasma. Indian J Dermatol Venereol Leprol. 2001;67(2):82-84.

29. Javaheri SM, Handa S, Kaur I, Kumar B. Safety and efficacy of glycolic acid facial peel in Indian women with melasma. Int J Dermatol. 2001;40(5):354-357.

30. Sarkar R, Kaur C, Bhalla M, Kanwar AJ. The combination of glycolic acid peels with a topical regimen in the treatment of melasma in dark-skinned patients: a comparative study. Dermatol Surg. 2002;28(9): 828-832; discussion 832.

31. Hurley ME, Guevara IL, Gonzales RM, Pandya AG. Efficacy of glycolic acid peels in the treatment of melasma. Arch Dermatol. 2002;138(12):1578-1582.

32. Sarkar R, Bansal S, Garg VK. Chemical peels for melasma in darkskinned patients. J Cutan Aesthet Surg. 2012;5(4):247-253.

33. Khunger N, Sarkar R, Jain RK. Tretinoin peels versus glycolic acid peels in the treatment of Melasma in dark-skinned patients. Dermatol Surg. 2004;30(5):756-760; discussion 760.

34. Kligman DE. Tretinoin peels versus glycolic acid peels. Dermatol Surg. 2004;30(12 Pt 2):1609.

35. Erbil H, Sezer E, Tastan B, Arca E, Kurumlu Z. Efficacy and safety of serial glycolic acid peels and a topical regimen in the treatment of recalcitrant melasma. J Dermatol. 2007;34(1):25-30.

36. Rendon M, Cardona LM, Bussear EW, BenitezAL, Colón LE, Johnson LA. Successful treatment of moderate to severe melasma with triple-combination cream and glycolic acid peels: a pilot study. Cutis. 2008;82(5):372-378.

37. Godse KV. Triple combination of hydroquinone, tretinoin and mometasone furoate with glycolic acid peels in melasma. Indian J Dermatol. 2009;54(1):92-93.

38. Godse KV, Sakhia J. Triple combination and glycolic acid peels in melasma in Indian patients. J Cosmet Dermatol. 2011;10(1): 68-69.

39. Kumari R, Thappa DM. Comparative study of trichloroacetic acid versus glycolic acid chemical peels in the treatment of melasma. Indian J Dermatol Venereol Leprol. 2010;76(4):447.
40. Puri N. Comparative study of $15 \%$ TCA peel versus $35 \%$ glycolic acid peel for the treatment of melasma. Indian Dermatol Online J. 2012;3(2):109-113.

41. Burns RL, Prevost-Blank PL, Lawry MA, Lawry TB, Faria DT, Fivenson DP. Glycolic acid peels for post inflammatory hyperpigmentation in black patients. A comparative study. Dermatol Surg. 1997;23(3):171-174; discussion 175.

42. Tse Y, Ostad A, Lee HS, et al. A clinical and histologic evaluation of two medium-depth peels. Glycolic acid versus Jessner's trichloroacetic acid. Dermatol Surg. 1996;22(9):781-786.

43. Piacquadio D, Dobry M, Hunt S, Andree C, Grove G, Hollenbach KA. Short contact $70 \%$ glycolic acid peels as a treatment for photodamaged skin. A pilot study. Dermatol Surg. 1996;22(5):449-452.

44. Newman N, Newman A, Moy LS, Babapour R, Harris AG, Moy RL. Clinical improvement of photoaged skin with $50 \%$ glycolic acid. A double-blind vehicle-controlled study. Dermatol Surg. 1996;22(5):455-460.

45. Moy LS, Murad H, Moy RL. Glycolic acid peels for the treatment of wrinkles and photoaging. J Dermatol Surg Oncol. 1993;19(3):243-246.

46. Sharad J. Combination of microneedling and glycolic acid peels for the treatment of acne scars in dark skin. J Cosmet Dermatol. 2011;10(4): 317-323.

47. Coleman WP III, Futrell JM. The glycolic acid trichloroacetic acid peel. J Dermatol Surg Oncol. 1994;20(1):76-80.

48. Moy LS. Superficial chemical peels with alpha-hydroxy acids. In: Robinson JK, Arndt KA, Le Boit PE, Wintroub BU, editors. Atlas of Cutaneous Surgery. Philadelphia, PA: WB Saunders; 1996:345-350.

49. Monheit GD. The Jessner's + TCA peel: a medium-depth chemical peel. J Dermatol Surg Oncol. 1989;15(9):945-950.

50. Monheit GD. How to select the appropriate peel for each patient. In: Rubin MG, Tung R, editors. Procedures in Cosmetic Dermatology Series: Chemical Peels. St Louis, MO: Elsevier; 2006:115-136.

51. Marrero GM, Katz BE. The new fluor-hydroxy pulse peel. A combination of 5-fluorouracil and glycolic acid. Dermatol Surg. 1998;24(9): 973-978.

52. Kempiak SJ, Uebelhoer N. Superficial chemical peels and microdermabrasion for acne vulgaris. Semin Cutan Med Surg. 2008;27(3): 212-220.

53. Briden E, Jacobsen E, Johnson C. Combining superficial glycolic acid (alpha-hydroxy acid) peels with microdermabrasion to maximize treatment results and patient satisfaction. Cutis. 2007; 79(1 Suppl Combining):13-16.

54. Fournier N, Fritz K, Mordon S. Use of nonthermal blue (405- to 420-nm) and near-infrared light (850- to 900-nm) dual-wavelength system in combination with glycolic acid peels and topical vitamin C for skin photo rejuvenation. Dermatol Surg. 2006;32(9): 1140-1146.

55. Sobhi RM, Sobhi AM. A single-blinded comparative study between the use of glycolic acid $70 \%$ peel and the use of topical nanosome vitamin $\mathrm{C}$ iontophoresis in the treatment of melasma. $J$ Cosmet Dermatol. 2012;11(1):65-71.

56. Rendon MI, Effron C, Edison BL. The use of fillers and botulinum toxin type A in combination with superficial glycolic acid (alpha-hydroxy acid) peels: optimizing injection therapy with the skin-smoothing properties of peels. Cutis. 2007;79(1 Suppl Combining):9-12.

57. Landau M. Combination of chemical peelings with botulinum toxin injections and dermal fillers. J Cosmet Dermatol. 2006;5(2):121-126.

58. Perić S, Bubanj M, Bubanj S, Jančić S. Side effects assessment in glycolic acid peelings in patients with acne type I. Bosn J Basic Med Sci. 2011;11(1):52-57.

59. Monheit GD, Chastain MA. Chemical peels. Facial Plast Surg Clin North Am. 2001;9(2):239-255.

60. Duffy DM. Avoiding complications. In: Rubin MG, Tung R, editors. Procedures in Cosmetic Dermatology Series: Chemical Peels. St Louis, MO: Elsevier; 2006:137-169.

61. Vishal B, Rao SS, Pavithra S, Shenoy MM. Contact urticaria to glycolic acid peel. J Cutan Aesthet Surg. 2012;5(1):58-59. 


\section{Publish your work in this journal}

Clinical, Cosmetic and Investigational Dermatology is an international, peer-reviewed, open access, online journal that focuses on the latest clinical and experimental research in all aspects of skin disease and cosmetic interventions. All areas of dermatology will be covered; contributions will be welcomed from all clinicians and

basic science researchers globally. This journal is indexed on CAS.

The manuscript management system is completely online and includes

a very quick and fair peer-review system, which is all easy to use. Visit http://www.dovepress.com/testimonials.php to read real quotes from published authors.

Submit your manuscript here: http://www.dovepress.com/clinical-cosmetic-and-investigational-dermatology-journal 